\title{
Transient reduction in erythrocyte membrane sialoglycoprotein $\beta$ associated with the presence of elliptocytes
}

\author{
G. L. Daniels, ${ }^{1}$ M. E. Reid, ${ }^{2}$ D. J. Anstee, ${ }^{3}$ K. M. Beattie ${ }^{4}$ And W. J. Judd ${ }^{5}{ }^{1}$ Medical Research Council \\ Blood Group Unit, Wolfson House, 4 Stephenson Way, London NW1 2HE, ${ }^{2}$ Department of Laboratory Medicine, \\ San Francisco General Hospital Medical Center, 1001 Potrero Avenue, San Francisco, CA 94110, U.S.A., \\ ${ }^{3}$ South Western Regional Transfusion Centre, Southmead Road, Bristol BS10 5ND, ${ }^{4}$ American Red Cross Blood \\ Services, Southeastern Michigan Region, 100 Mack Avenue, Box 33351, Detroit, Michigan 48232, U.S.A., and \\ ${ }^{5}$ University of Michigan Hospitals, Department of Pathology, 1500 E. Medical Center Drive, Ann Arbor. \\ Michigan 48109-0054, U.S.A.
}

Received 22 January 1988; accepted for publication 22 June 1988

\begin{abstract}
Summary. Erythrocyte membranes from an anaemic patient receiving gold therapy for rheumatoid arthritis had reduced $\beta$-sialoglycoprotein ( $\beta$-SGP) content but normal expression of sialoglycoproteins $\alpha, \delta$ and $\gamma$. Elliptocytes were present in the peripheral blood. The serum of the patient contained anti- $\beta$ SGP which did not appear to bind to her own cells. It reacted with all erythrocytes apart from $\beta$-SGP deficient Leach phenotype cells. The antibody was inhibited by purified $\beta$ SGP from normal red cells, bound to $\beta$-SGP on immunoblots and also reacted with the abnormal $\beta$-related-SGP in erythrocyte membranes of both the Gerbich type and Yus type of
\end{abstract}

Gerbich negative. Two years later the patient was no longer anaemic, no elliptocytes were seen in her peripheral blood film and her erythrocyte membranes had normal $\beta$-SGP content. Antibody was no longer present in her serum and antibody from the earlier sample now reacted with the patient's erythrocytes.

Erythrocyte membrane $\beta$-SGP is known to be important in the maintenance of normal cell shape. It is likely that the transient occurrence of elliptocytes in the patient resulted from the concurrent temporary reduction in $\beta$-SGP content of her erythrocyte membranes.
The human erythrocyte membrane contains at least four different sialoglycoproteins (SGPs), $\alpha, \beta, \gamma$ and $\delta$ (for review see Anstee \& Tanner, 1986). There is now substantial evidence that two of these SGPs, $\beta$ (syn: glycoconnectin, glycophorin $\mathbf{C}$ ) and $\gamma$, are directly involved in the maintenance of erythrocyte shape (Mueller \& Morrison, 1981). Gerbich (Ge) is a blood group comprising at least two erythrocyte antigens of very high frequency in most populations (for review see Race \& Sanger, 1975; Reid, 1986). There are at least three Ge-negative phenotypes, the original Gerbich type (Ge:-2,-3), the Yus type (Ge:-2,3) and the Leach phenotype (also Ge:-2,-3). Unlike the other two Ge-negative types, the Leach phenotype is characterized by elliptocytosis. Leach phenotype erythrocytes lack SGPs $\beta$ and $\gamma$ in the cell membrane, fail to react with murine monoclonal antibodies directed at a portion of $\beta$-SGP (Anstee et al, 1984a; Daniels et al, 1986; Reid et al, 1987c), and have marked reduction in

Correspondence: Dr G. L. Daniels, MRC Blood Group Unit, Wolfson House, 4 Stephenson Way, London NW1 $2 \mathrm{HE}$. both membrane mechanical stability and membrane deformability, providing clear evidence of an important functional role for $\beta$ and $\gamma$ SGPs in normal erythrocytes (Reid et al, 1987b).

Recently, Beattie \& Sigmund (1987) described a severely anaemic patient with elliptocytosis who had received gold therapy for rheumatoid arthritis and who had produced a Gelike antibody. In this paper we report a detailed study of the erythrocyte membranes of this patient and of the immunochemical specificity of her antibody. The results show that the antibody may have been anti- $\beta$-SGP and that elliptocytosis, which was transient, was associated with a transient reduction in the membrane content of $\beta$-SGP. We believe this to be the first report of an acquired $\beta$-SGP deficiency.

\section{MATERIALS AND METHODS}

Materials. Blood samples from the patient were collected during her aplastic crisis (October 1982 and November 1982) and during remission (October 1984). For long-term 
storage, the red cells were stored in liquid nitrogen and the plasma samples stored at $-20^{\circ} \mathrm{C}$.

Human alloantibodies were gifts from a variety of sources, as were erythrocytes from Ge-negative donors. For immunoblotting and haemagglutination tests against sialidasetreated erythrocytes, and against $\mathrm{Tn}$ and Cad erythrocytes, the human antibodies were purified as described by Tanner $e t$ al (1988). A rabbit antiserum, reactive with the cytoplasmic portions of SGPs $\beta$ and $\gamma$, was prepared as described (Reid $e t a l$, 1987a). Purified $\beta$-SGP was prepared as described by Reid $e t$ al (1987a). Endoglycosidase $\mathrm{F} /$ peptidyl- $N$-glycosidase $\mathrm{F}$ (Endo F) preparations were prepared and used as described in Tanner et al (1988).

Serological methods. Standard serological methods were used. For antiglobulin tests polyspecific anti-human globulin was used for human antibodies, and anti-mouse IgG (provided by Dr R. W. Knowles) for murine monoclonal antibodies. Red cells were treated with proteases and sialidase as described previously (Anstee et al, 1984b). For haemagglutination inhibition tests a volume of serum was incubated with an equal volume of inhibitor for $20 \mathrm{~min}$ at room temperature before adding a volume of the appropriate erythrocyte suspension followed by an indirect antiglobulin test.

Biochemical methods. Sodium dodecyl sulphate (SDS)polyacrylamide gel electrophoresis of erythrocyte membranes was carried out on gels containing $10 \%$ acrylamide with a $3 \%$ overlay using the method of Laemmli (1970). The protein concentration of erythrocyte membranes was determined by the method of Lowry et al (1951) in the presence of $0 \cdot 1 \%$ SDS. Periodic acid Schiff's base (PAS) stained gels were scanned at $550 \mathrm{~nm}$ on a Beckman scanning spectrophotometer and the areas calculated using a lowest valley integration method. The relative concentration of spectrin. protein $4 \cdot 1$ and band 3 was determined by pyridine elution (Agre et al, 1985). Immunoblotting was as described by Mallinson et al (1986), except that $5 \%(\mathrm{w} / \mathrm{v})$ bovine milk powder in phosphate-buffered saline (PBS) at $\mathrm{pH} 7 \cdot 4$ was used as the blocking agent. Binding of antibodies was detected using peroxidase-conjugated goat anti-mouse IgG, anti-human IgG or anti-rabbit IgG (BioRad, Richmond, Calif.).

\section{RESULTS}

Serological results on the erythrocytes of the patient

The following findings were obtained on the patient's erythrocytes during the time antibody was present in her serum (1982 sample). They reacted with six examples of alloanti-Ge3 (sera of Gerbich or Leach type individuals) and 29 examples of alloanti-Ge2 (sera of Yus type and some Gerbich or Leach type individuals). Titration of Ge antisera with the patient's cells, and tests both before and after treatment of the cells with proteases, showed that the patient's cells expressed normal amounts of Ge antigens as determined by human alloantibodies and that their $\mathrm{Ge}$ antigens, like those of normal erythrocytes, were sensitive to treatment with papain or trypsin.
When the anti- $\beta$-SGP monoclonal antibodies GERO (Daniels et al, 1983) and BRIC 10 (Anstee et al, 1984a, b) were used the patient's cells behaved like most Ge-negative cells: they were not agglutinated by the antibodies directly but reacted strongly by the antiglobulin test. After trypsin treatment the patient's cells did not react with the monoclonal antibodies by either method. In titrations with the monoclonal antibodies by the antiglobulin method, the patient's cells gave similar or only slightly lower scores than did Ge-positive control cells.

Ge-negative erythrocytes of both Gerbich and Leach types, but not of the Yus type, usually show reduced strength of expression of Kell and para-Kell blood group antigens (Daniels, 1982: Anstee et al, 1984a, b: Daniels et al, 1986; Reid et al, 1987c). The patient's erythrocytes were $\mathrm{K}-\mathrm{k}+$ $\mathrm{Kp}(\mathrm{a}-\mathrm{b}+\mathrm{c}-) \mathrm{Js}(\mathrm{a}-\mathrm{b}+) \mathrm{K}: 11,-17 \mathrm{Ul}(\mathrm{a}-) \mathrm{Ku}+\mathrm{K}: 12$, $13,18,22$. No weakness was apparent in the expression of any of the high frequency Kell or para-Kell antigens. The patient's cells were not $T$ active as determined by testing with Arachis hypogaea lectin.

In 1984, when antibody could no longer be detected in the patient's serum, her erythrocytes were Ge-positive as before but now resembled other Ge-positive erythrocytes by being agglutinated directly and indirectly by monoclonal anti- $\beta$ SGP.

\section{The erythrocyte membranes}

Erythrocyte membranes prepared from patient samples collected in 1982 and 1984, and adjusted to the same protein concentration, were analysed by SDS-PAGE with subsequent PAS staining. The results demonstrated normal staining of bands containing SGPs $\alpha, \delta$ and $\gamma$ in both samples. However, whilst normal staining of $\beta$-SGP was apparent in the 1984 sample, markedly reduced staining of $\beta$-SGP was evident in the 1982 sample (Fig la). Quantitation of the staining intensity of the $\beta$-SGP band in the 1982 sample by densitometry indicated that $\beta$ was about $35 \%$ of normal levels. When the gels were stained for protein with Coomassie blue, no differences from normal were observed with membranes from either 1982 or 1984 samples (data not shown). The ratios of spectrin : band $4 \cdot 1$ : band 3 were within normal limits in these samples.

The marked reduction in $\beta$-SGP was again apparent by immunoblotting with monoclonal anti- $\beta$-SGP (BRIC 10) (Fig 1e). When immunoblotting was carried out using a human alloanti-Ge3, which recognizes an extracellular antigenic determinant common to both $\beta$ and $\gamma$ SGPs (Reid $e t$ al, 1987a), reduced staining of $\beta$-SGP was again apparent in the 1982 sample, while staining of $\gamma$-SGP was comparable to normal control samples (Fig $1 \mathrm{j}$ ). Normal $\gamma$-SGP levels were also noted using human alloanti-Ge2 (Fig 1h).

Since both the PAS stain and the anti- $\beta$-SGP and anti$\beta+\gamma-$ SGP reagents are dependent on the presence of sialic acid on $\beta$-SGP for reactivity (Anstee et al, 1984b; Reid $e$ t al, $1987 \mathrm{a})$, the possibility existed that the apparent reduction of $\beta$-SGP in the 1982 sample was the result of a glycosylation abnormality, rather than a total reduction in $\beta$-SGP content. Immunoblots were therefore carried out using a rabbit antiserum rendered specific for the cytoplasmic portions of 


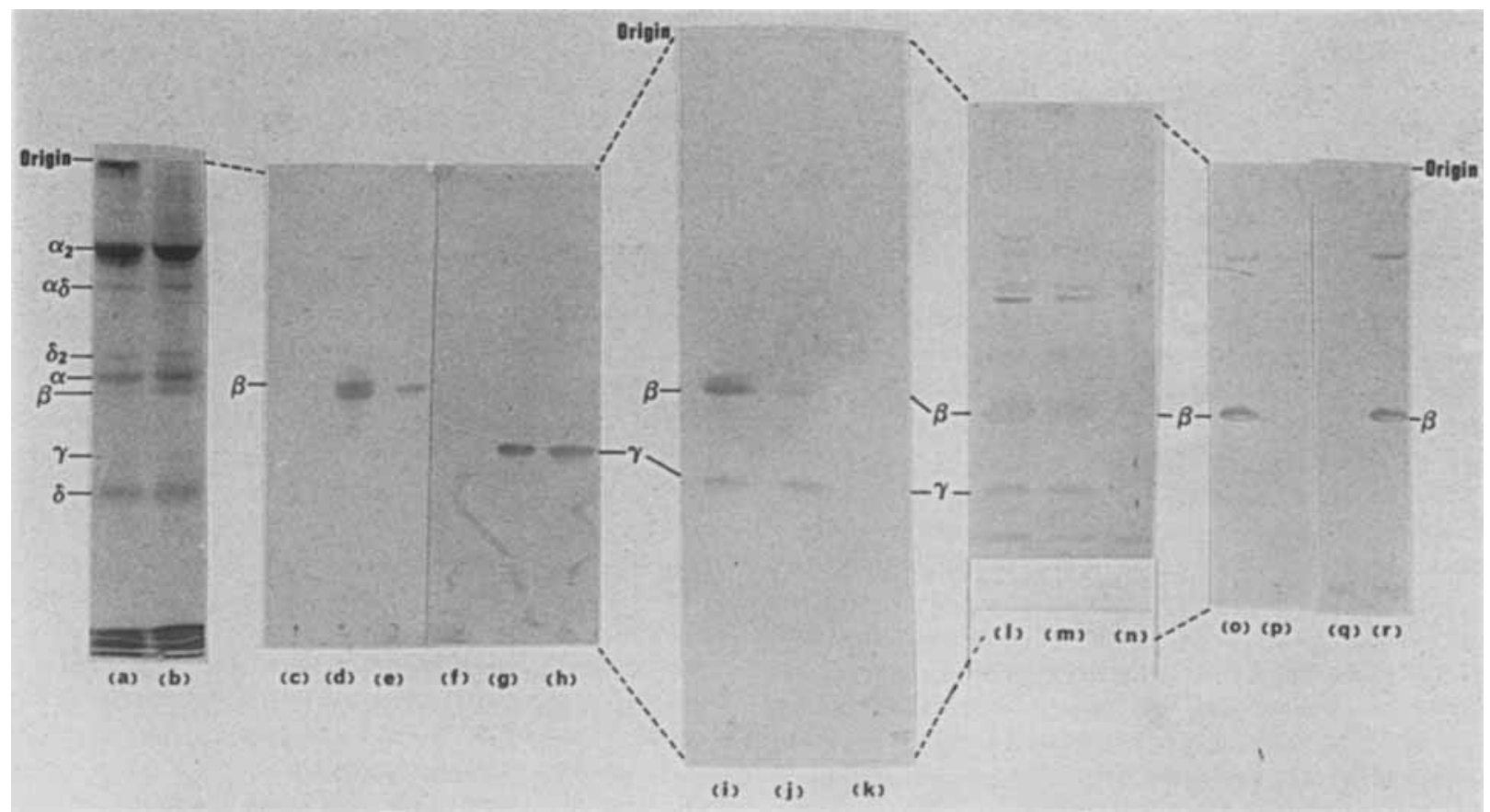

Fig 1. SDS-polyacrylamide gel electrophoresis of erythrocyte membranes of the patient and determination of the specificity of the patient's serum antibody. Tracks a and b: SDS-polyacrylamide gel electrophoresis of erythrocyte membranes from the patient (a, 1982 sample) and normal erythrocyte membranes (b) with subsequent PAS staining. Tracks c-e: Immunoblotting with murine monoclonal antibody BRIC 10, (c) membranes of Leach phenotype, (d) normal membranes, (e) membranes of patient (1982 sample). Tracks f-h: Immunoblotting with human alloanti-Ge2 (WHA), (f) Leach phenotype membranes, (g) normal membranes, (h) membranes of patient (1982 sample). Tracks i-k: Immunoblotting with human alloanti-Ge3 (44017), (i) normal membranes, (j) membranes of patient (1982 sample), (k) membranes of Leach phenotype. Tracks I-n: Immunoblotting with rabbit antibody to the cytoplasmic portions of SGPs $\beta$ and $\gamma$. (I) normal membranes. (m) membranes of patient (1982 sample), (n) membranes of Leach phenotype. Tracks o-r: Immunoblotting with antibody from the serum of the patient. (o), (r) normal membranes, (p) membranes of Leach phenotype, (q) membranes of patient (1982 sample).

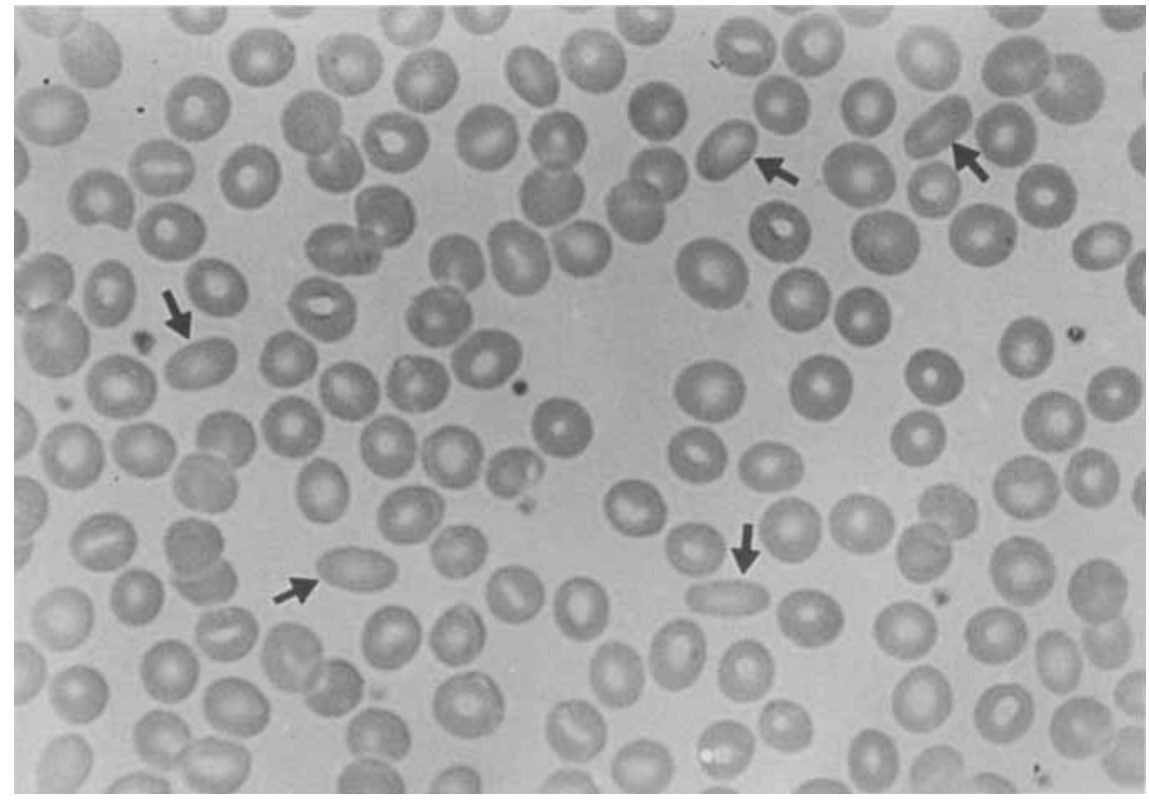

Fig 2. Morphology of erythrocytes from the patient. Photomicrograph of a smear of blood from the patient taken into EDTA. The sample is representative of the blood morphology of the patient between June 1982 and December 1983. 
SGPs $\beta$ and $\gamma$. The results again showed reduced staining of $\beta$-SGP in the 1982 sample, although the reduction was not so marked as that seen with the sialic acid dependent antibodies (Fig 1m).

\section{The specificity of the antibody in the serum of the patient}

The antibody in the serum of the 1982 sample behaved as an alloantibody: it could not be absorbed with the patient's own cells that had also been collected in 1982. However, the antibody was not present in serum collected in 1984 , and the original antibody reacted with the patient's erythrocytes from the 1984 sample both by the antiglobulin test and weakly by direct agglutination.

The antibody agglutinated Ge-positive cells and reacted by the antiglobulin test. It behaved like the monoclonal antibodies GERO and BRIC 10 in failing to agglutinate Ge negative erythrocytes of both Gerbich (six examples) and Yus (four examples) types in direct agglutination tests whilst still reacting with cells of either phenotype in antiglobulin tests; like the two monoclonals, the patient's antibody failed to react with Leach phenotype cells (three examples) in either direct or indirect agglutination tests. The antibody could not be absorbed by Leach phenotype cells.

The patient's serum failed to react with Ge-positive erythrocytes treated with papain, ficin, trypsin or pronase but did react with $\alpha$-chromotrypsin-treated erythrocytes. Like monoclonal anti- $\beta$-SGP, the patient's antibody did not react with trypsin-treated Yus type cells but did react, both by direct and indirect agglutination, with trypsin-treated Gerbich type cells.

Purified antibody from the patient did not agglutinate sialidase-treated erythrocytes either directly or indirectly but did react with Cad 1 and Tn erythrocytes in indirect agglutination tests, although the reactions were weaker than with normal erythrocytes. In haemagglutination inhibition tests, purified $\beta$-SGP from normal erythrocytes completely inhibited the patient's antibody.

By immunoblotting, the patient's purified antibody was clearly specific for $\beta$-SGP and did not react with $\gamma$-SGP (Fig 10, r). The antibody also bound to the abnormal $\beta$-SGPrelated sialoglycoproteins found in membranes from Gerbich type and from Yus type erythrocytes (data not shown). No reactivity was observed with Leach phenotype membranes which lack both $\beta$-SGP and $\gamma$-SGP and do not contain the abnormal $\beta$-SGP-related components of Ge or Yus-type Gerbich-negative cells (Fig 1p), or with membranes of autologous red cells collected in 1982 (Fig 1q). With membranes derived from Endo F-treated normal erythrocytes, the patient's antibody reacted with the band corresponding to $\beta$-SGP after removal of the $N$-glycosidically-linked oligosaccharide (data not shown). The antibody also reacted by immunoblotting with the abnormal $\beta^{\text {wb }}$ SGP of $W b$ positive erythrocytes (data not shown) which are believed to have abnormal glycosylation (Reid et al, 1985). Collectively, these results imply that the reactivity of the patient's antibody did not depend on the presence of the $N$-glycosidically linked oligosaccharide normally found on $\beta$-SGP.

\section{Erythrocyte morphology}

Elliptocytes were present in peripheral blood films from the patient between June 1982 and December 1983 (Fig 2). No elliptocytes have been observed in subsequent blood films.

\section{DISCUSSION}

The patient reported here produced a Ge-like blood group antibody which behaved as an alloantibody. Two years later, the antibody was no longer detectable in the patient's serum and her erythrocytes reacted with the antibody in her previous serum sample.

We have shown that the patient's antibody is specific for $\beta$-SGP, a molecule which has been well characterized (Dahr $e t$ al, 1982; Colin et al, 1986). The availability of antibodies to different antigenic determinants on both the extracellular and intracellular portions of $\beta$-SGP allowed us to obtain evidence that, at the time when the anti- $\beta$-SGP was detected, the content of $\beta$-SGP in the patient's erythrocyte membranes was markedly reduced. That is, there was a reduction in the amount of the whole molecule in the membrane, and not simply a change which affected an extracellular region of the molecule involving a particular epitope, or group of epitopes. However, the patient's red cells failed to adsorb her $\beta$-SGPspecific antibody, suggesting that this antibody was demonstrating some subtle qualitative difference between the residual $\beta$-SGP and normal $\beta$-SGP, not recognized by the murine monoclonal or rabbit antibodies. The $\gamma$-SGP content of the patient's erythrocyte membranes appeared normal. This apparently selective effect on $\beta$-SGP. but not the structurally related $\gamma$-SGP (Reid et al, 1987a), is difficult to interpret.

SGPs $\beta$ and $\gamma$ have a well-defined function, viz maintenance of normal erythrocyte shape, deformability and membrane mechanical stability through interaction of the cytoplasmic portion of the molecule with the membrane skeleton (Mueller \& Morrison, 1981; Anstee et al, 1984a, b; Reid et al, 1987b). Total absence of SGPs $\beta$ and $\gamma$, as occurs in erythrocytes of the Leach phenotype, is associated with elliptocytosis (Anstee et al, 1984a; Daniels et al, 1986; Reid et al, $1987 \mathrm{c}$ ). We suggest, but cannot prove, that the transient occurrence of elliptocytes in the peripheral blood of the patient described here, which occurred during the period when the $\beta$-SGP content of her erythrocyte membranes was reduced and disappeared when the $\beta$-SGP content returned to normal, resulted from the reduced $\beta$-SGP content of her erythrocyte membranes. The anaemia, however, did not correlate with the abnormal serological and biochemical findings and probably resulted from the gold therapy. It is hoped that this report may lead to further examples of this phenomenon being recognized.

\section{ACKNOWLEDGMENTS}

We wish to thank the following: Dr J. Hoschner and Ms J. McGraw, Harper Hospital, Detroit, Michigan, U.S.A., for blood samples from the patient; Mr G. Rowe, Cardiff Regional Transfusion Centre, Wales, for Wb-positive erythrocytes; Dr J.-P. Cartron, Inserm Unite 76. Paris, France, for Cad 
erythrocytes; Dr G. Inglis and Dr C. Gabra, Glasgow and West of Scotland Transfusion Service, Carluke, Scotland, and Mr M. L. Beck, Community Blood Center of Greater Kansas City, Kansas, U.S.A., for Tn erythrocytes; Dr M. J. A. Tanner, University of Bristol, England, for making available the Endo F-treated cells.

\section{REFERENCES}

Agre, P., Casella, J-F., Zinkham, W.H., McMillan, C. \& Bennet, V. (1985) Partial deficiency of erythrocyte spectrin in hereditary spherocytosis. Nature, 314, 380-383.

Anstee, D.J., Parsons, S.F., Ridgwell. K., Tanner, M.J.A., Merry, A.H., Thomson, E.E., Judson, P.A., Johnson, P., Bates, S. \& Fisher, I.D. (1984a) Two individuals with elliptocytic red cells apparently lack three minor erythrocyte membrane sialoglycoproteins. Biochemical Journal, 218, 615-619.

Anstee, D.J., Ridgwell, K., Tanner, M.J.A., Daniels, G.L. \& Parsons, S.F. (1984b) Individuals lacking the Gerbich blood group-antigen have alterations in the human erythrocyte membrane sialoglycoproteins $\beta$ and $\gamma$. Biochemical Journal, 221, 97-104.

Anstee, D.J. \& Tanner, M.J.A. (1986) Structure and functions of the red cell membrane sialoglycoproteins. British Journal of Haematology, 64, 211-215.

Beattie, K.M. \& Sigmund, K.E. (1987) A Ge-like autoantibody in the serum of a patient receiving gold therapy for rheumatoid arthritis. Transfusion, 27, 54-57.

Colin, Y., Rahuel, C., London, J., Romeo, P-H., D'Auriol, L., Galibert, F. \& Cartron, J-P. (1986) Isolation of cDNA clones and complete amino acid sequence of human erythrocyte glycophorin C. Journal of Biological Chemistry. 261, 229-233.

Dahr, W., Beyreuther, K., Kordowicz, M. \& Kruger, J. (1982) Nterminal amino acid sequence of sialoglycoprotein D (glycophorin C) from human erythrocyte membranes. European Journal of Biochemistry, 125, 57-62.

Daniels, G.L. (1982) Studies on Gerbich negative phenotypes and Gerbich antibodies. (Abstract). Transfusion, 22, 405.

Daniels, G.L., Shaw, M.A., Judson, P.A., Reid, M.E., Anstee, D.J., Colpitts, P., Cornwall, S., Moore, B.P.L. \& Lee, S. (1986) A family demonstrating inheritance of the Leach phenotype: a Gerbichnegative phenotype associated with elliptocytosis. Vox Sanguinis, 50, 117-121.

Daniels, G.L., Banting. G. \& Goodfellow, P. (1983) A monoclonal
Sialoglycoprotein $\beta$ and Elliptocytosis

antibody related to the human blood group Gerbich. Journal of Immunogenetics, 10, 103-105.

Laemmli, U.K. (1970) Cleavage of structural proteins during the assembly of the head of bacteriophage T4. Nature, 227, 680-685.

Lowry, O.H., Rosenborough, N.J., Farr, A.L. \& Randell, R.J. (1951) Protein measurement with the Folin phenol reagent. Journal of Biological Chemistry, 193, 265-175.

Mallinson, G., Martin, P.G., Anstee, D.J., Tanner, M.J.A., Merry, A.H., Tills, D. \& Sonneborn, H.H. (1986) Identification and partial characterization of the human erythrocyte membrane component(s) that express the antigens of the LW blood-group system. Biochemical Journal, 234, 649-652.

Mueller, T.J. \& Morrison, M. (1981) Glycoconnectin (PAS 2), a membrane attachment site for the human erythrocyte cytoskeleton. Erythrocyte Membranes 2: Recent Clinical and Experimental Advances, pp. 95-112. Liss, New York.

Parsons, S.F., Judson, P.A. \& Anstee, D.J. (1982) BRIC 18: a monoclonal antibody with a specificity related to the Kell blood group system. Journal of Immunogenetics, 9, 377-380.

Race. R.R. \& Sanger, R. (1975) Blood Groups in Man. Blackwell Scientific Publications, Oxford.

Reid, M.E. (1986) The Gerbich blood group antigens: a review. Medical Laboratory Sciences, 43, 177-182.

Reid, M.E., Anstee, D.J., Tanner, M.J.A., Ridgwell, K. \& Nurse, C.T. (1987a) Structural relationships between human erythrocyte sialoglycoproteins $\beta$ and $\gamma$ and abnormal sialoglycoproteins found in certain rare human erythrocyte variants lacking the Gerbich blood group antigen(s). Biochemical Journal, 244, 123-128.

Reid, M.E., Chasis, J.A. \& Mohandas, N. (1987b) Identification of a functional role for erythrocyte sialoglycoproteins. Blood, 69, 1068-1072.

Reid, M.E., Matynewycz, M.A., Walford, F.E., Crawford, M.N. \& Miller, L.H. (1987c) Leach type Ge- red cells and elliptocytosis. Transfusion, 27, 213-214.

Reid, M.E., Shaw, M.A., Rowe, G., Anstee, D.J. \& Tanner, M.J.A. (1985) Abnormal minor human erythrocyte membrane sialoglycoprotein $(\beta)$ in association with the rare blood group antigen Webb (Wb). Biochemical Journal, 232, 289-291.

Tanner, M.J.A., Anstee, D.J., Mallinson, G., Ridgwell, K., Martin, P.G., Avent, N.D. \& Parsons, S.F. (1988) Effect of endoglycosidase $\mathrm{F} /$ peptidyl N-glycosidase $\mathrm{F}$ preparations on the surface components of the human erythrocyte. Carbohydrate Research, 178, 203-212. 